(1)

CrossMark

\title{
Remote monitoring of oxygen saturation in individuals with COVID-19 pneumonia
}

\author{
To the Editor:
}

Coronavirus disease 2019 (COVID-19), an illness caused by severe acute respiratory syndrome coronavirus 2 (SARS-CoV-2) infection, has spread rapidly worldwide, resulting in significant mortality and placing major strain on healthcare systems. Although the clinical course is variable, one in five patients will require hospitalisation for management, with older age and the presence of comorbidities increasing the risk of more severe disease [1-3]. The median time from first onset of symptoms to development of acute respiratory distress syndrome in those who progress to severe disease is estimated to be 8.0 days [4]. Currently, there is an increased pressure on acute hospital-based care and a corresponding demand for novel solutions to address this. One approach is to utilise telemedicine to facilitate efficient and safe discharge from acute hospitals. Due to the highly contagious nature of SARS-CoV-2, routine or urgent clinical follow-up is difficult within the primary care or community settings. Hence, the ability to remotely monitor symptoms and vital signs in mild-to-moderate cases may be one mechanism to alleviate the pressures on healthcare systems, and we present here some initial data on one such approach.

Over a 1-month period, 193 COVID-19-positive patients were admitted to St Vincent's University Hospital, an 836-bed tertiary referral centre in Dublin, Ireland. All patients were offered enrolment into the All-Ireland Infectious Diseases Cohort Study, a multicentre, prospective cohort recruiting patients accessing clinical care related to infectious diseases presenting to several affiliated hospitals in Ireland. A proportion of patients that had pulmonary infiltrates on chest radiographs and did not need or no longer required supplemental oxygen were provided with a NoninConnect 3230 Bluetooth Smart Pulse Oximeter (Nonin Medical Inc., Plymouth, MN, USA) upon discharge from hospital. The oximeters were linked to a mobile application specifically developed to monitor oxygen saturation and self-reported breathlessness in individuals with COVID-19 (patientMpower Ltd, Dublin, Ireland). All data were encrypted and sent to a secure cloud database that was accessible only to members of the COVID-19 monitoring team. The mobile application sent an automatic prompt to check oxygen saturations at rest and not following exertion, four times daily for 14 days after discharge from hospital, and patients could enter additional data/ measurements at will. A prompt also asked if the patient felt breathless. If the patient selected "yes", then they were asked to use a visual analogue scale to measure this from 1-10; however, most patients did not report being breathless. If oxygen saturation measurements were $\leqslant 94 \%$, an alert was generated, resulting in an SMS text message being sent to the monitoring team, composed of respiratory physicians and nurse specialists. Following an alert, the person was contacted by the team and if there was persistent hypoxia, a deterioration in symptoms, or any other cause for concern, they were instructed to present to the hospital for an assessment.

In the reported period, 26 patients were suitable for home monitoring as part of clinical care, and 18 patients that utilised the remote monitoring platform consented for their data to be used. Table 1 outlines the demographic and clinical characteristics of this cohort during their initial admission. Over this period, 917 data-points regarding peripheral capillary oxygen saturation $\left(S_{\mathrm{pO}_{2}}\right)$ were generated, resulting in a total of 51 alerts (5.5\% of all $S_{\mathrm{pO}_{2}}$ measurements). Patients were instructed to record saturations for 14 days, but several patients recorded for a shorter or longer period and the median time spent on the monitor was 12 days. These alerts resulted in five reassessments and four individuals were readmitted to hospital as outlined. These patients were readmitted 2, 3, 8 and 10 days after their initial discharge from hospital. There were no apparent differences in the initial admission profiles of the patients requiring

@ERSpublications

Remote monitoring of oxygen saturation in cases of COVID-19 pneumonia may facilitate discharge, relieving burden on bed demand and allowing safe follow-up for this disease in which the sequelae are unknown https://bit.ly/3cTXnZU

Cite this article as: O'Carroll O, MacCann R, O'Reilly A, et al. Remote monitoring of oxygen saturation in individuals with COVID-19 pneumonia. Eur Respir J 2020; 56: 2001492 [https://doi.org/10.1183/ 13993003.01492-2020]. 
TABLE 1 Demographic and clinical characteristics of the remote monitoring cohort

\begin{tabular}{|c|c|c|c|}
\hline & Whole cohort & No readmission & Readmission \\
\hline Patients n & 18 & 14 & 4 \\
\hline Age years & $48(41-60)$ & $46(37-57)$ & $56(48-63)$ \\
\hline Female & 6 (33.3) & $3(21.5)$ & $3(75)$ \\
\hline Current smoker & $1(5.5)$ & $1(7.1)$ & $0(0)$ \\
\hline Ex-smoker & $5(2.7)$ & 4 (28.5) & $1(25)$ \\
\hline Required supplemental oxygen & $16(89)$ & $13(92.8)$ & $3(75)$ \\
\hline ICU admission & $4(22)$ & $4(28.5)$ & $0(0)$ \\
\hline Mechanical ventilation & $0(0)$ & $0(0)$ & $0(0)$ \\
\hline NIV & $5(27)$ & $5(35.7)$ & $0(0)$ \\
\hline Received hydroxychloroquine and azithromycin & $13(72)$ & 10 (71.4) & $3(75)$ \\
\hline Time on home monitor days & $12(9.75-18)$ & $12.5(10-18)$ & $5.5(2-10)$ \\
\hline$S_{\mathrm{pO}_{2}}$ for alerts $\%$ & $92(92-93)$ & $93(92-93)$ & $91.5(89.5-92)$ \\
\hline
\end{tabular}

Data are presented as median (interquartile range) or $\mathrm{n}(\%)$, unless otherwise stated. Data for readmission patients relates to the initial hospital admission only. ICU: intensive care unit; NIV: noninvasive ventilation; $\mathrm{S}_{\mathrm{pO}_{2}}$ : peripheral capillary oxygen saturation.

readmission apart from a shorter initial length of stay. The median $S_{\mathrm{pO}_{2}}$ of these alerts was $91 \%$ and the lowest $\mathrm{S}_{\mathrm{pO}_{2}}$ recorded was $82 \%$. Three patients that were readmitted had progressive infiltrates and worse hypoxia related to COVID-19, and one patient had pneumonia unrelated to their previous radiographic changes of COVID-19, which had resolved on initial discharge. None of the readmitted patients required noninvasive or invasive ventilation during readmission.

The utility of remote monitoring systems has been an increasingly studied subject, and there is growing evidence that remote monitoring can facilitate more streamlined approaches to observational studies and the delivery of patient care, especially in pulmonary diseases [5, 6]. We believe that this system contributed positively to patient confidence and reassurance regarding discharge from hospital, especially with COVID-19 being a relatively unknown entity, and in a climate of significant concern and anxiety about their condition. However, given the scope of this report, patient confidence or satisfaction was not formally assessed. Nevertheless, in this cohort of patients, there was excellent adherence, which can be a surrogate for patient acceptance [7]. Indeed, the median number of measurements per day was 3.9 in the group which did not require readmission and 5.7 in those readmitted, both higher than $97 \%$ adherence [7]. This may have been aided by the minimal inputs needed and the acute aspect of this illness compared to the difficulties and challenges noted in compliance with remote monitoring in chronic diseases $[8,9]$.

The use of modern mobile technology to specifically monitor individuals with COVID-19 allowed us to facilitate discharge in patients with mild-to-moderate disease in a safe and appropriate manner. While in this study a relatively high number of patients were readmitted, we believe this reflects the severity of the initial illness. Only patients who required hospitalisation and had pulmonary infiltrates were enrolled to home monitoring. Potentially, those with milder COVID-19 could be monitored for any deterioration, and this could indeed identify patients deteriorating at home. However, we did not examine this. Moreover, the discharge strategy we employed relieved demand for hospital beds during this period of significantly increased demand. During the reported 1-month period our hospital was operating at surge capacity with $125 \%$ occupancy of the intensive care unit, utilising other wards/areas to mechanically ventilate patients. The patients who utilised this monitoring system were discharged from an acute care hospital system under strain in a manner that contributed to increased bed availability without compromising safe patient care. Use of remote monitoring ensured early recognition of acute deterioration in recently discharged patients and enabled reassessment and readmission when necessary. This type of approach has the potential to conserve hospital resources for those most in need, while simultaneously enabling early identification of those who deteriorate acutely and require urgent assessment. 
Orla O'Carroll ${ }^{1}$, Rachel MacCann ${ }^{2}$, Aoife O'Reilly ${ }^{1,3}$, Eleanor M. Dunican ${ }^{1,3}$, Eoin R. Feeney ${ }^{2,4}$, Silke Ryan $\oplus^{1,3}$, Aoife Cotter ${ }^{4}$, Patrick W. Mallon ${ }^{2,4}$, Michael P. Keane ${ }^{1,3}$, Marcus W. Butler ${ }^{1,3}$ and Cormac McCarthy ${ }^{1,3}$

${ }^{1}$ Dept of Respiratory Medicine, St Vincent's University Hospital, Dublin, Ireland. ${ }^{2}$ Dept of Infectious Diseases, St Vincent's University Hospital, Dublin, Ireland. ${ }^{3}$ School of Medicine, University College Dublin, Dublin, Ireland. ${ }^{4}$ Centre for Experimental Pathogen Host Research (CEPHR), University College Dublin, Dublin, Ireland.

Correspondence: Cormac McCarthy, Education and Research Centre, University College Dublin, St Vincent's University Hospital, Dublin 4, Ireland. Email: Cormac.McCarthy@UCD.ie

Received: 30 April 2020 | Accepted after revision: 9 June 2020

Conflict of interest: None declared.

\section{References}

1 Wu Z, McGoogan JM. Characteristics of and important lessons from the coronavirus disease 2019 (COVID-19) outbreak in China: summary of a report of 72314 cases from the Chinese Center for Disease Control and Prevention. JAMA 2020; 323: 1239-1242.

2 Grasselli G, Zangrillo A, Zanella A, et al. Baseline characteristics and outcomes of 1591 patients infected with SARS-CoV-2 admitted to ICUs of the Lombardy region, Italy. JAMA 2020; 323: 1574-1581.

3 Zhou F, Yu T, Du R, et al. Clinical course and risk factors for mortality of adult inpatients with COVID-19 in Wuhan, China: a retrospective cohort study. Lancet 2020; 395: 1054-1062.

4 Wang $\mathrm{D}, \mathrm{Hu} \mathrm{B}, \mathrm{Hu}$ C, et al. Clinical characteristics of 138 hospitalized patients with 2019 novel coronavirus-infected pneumonia in Wuhan, China. JAMA 2020; 323: 1061-1069.

5 Heaney LG, Busby J, Bradding P, et al. Remotely monitored therapy and nitric oxide suppression identifies nonadherence in severe asthma. Am J Respir Crit Care Med 2019; 199: 454-464.

6 Moor CC, Mostard RLM, Grutters JC, et al. Home monitoring in patients with idiopathic pulmonary fibrosis: a randomized controlled trial. Am J Respir Crit Care Med 2020; 202: 393-401.

7 Lang C, Voigt K, Neumann R, et al. Adherence and acceptance of a home-based telemonitoring application used by multi-morbid patients aged 65 years and older. J Telemed Telecare 2020; in press [https://doi.org/10.1177/ 1357633X20901400].

8 Rosenfeld LE, Patel AS, Ajmani VB, et al. Compliance with remote monitoring of ICDS/CRTDS in a real-world population. Pacing Clin Electrophysiol 2014; 37: 820-827.

9 Milan Manani S, Rosner MH, Virzi GM, et al. Longitudinal experience with remote monitoring for automated peritoneal dialysis patients. Nephron 2019; 142: 1-9. 